\title{
Sintonización de controladores PID por medio de un algoritmo genético con fertilización in vitro aplicado a un convertidor multicelular
}

\author{
J. F. García-Mejía ${ }^{1,2}$, A. A. Flores-Fuentes ${ }^{1}$, J. C. Ambriz-Polo ${ }^{3}$, L. A González-Escobar ${ }^{4}$, \\ C. E. Torres-Reyes ${ }^{1}$, E. E. Granda Gutiérrez ${ }^{1}$ \\ ${ }^{1}$ Universidad Autónoma del Estado de México, CUAEM Atlacomulco, \\ Estado de México, México \\ ${ }^{2}$ Centro Panamericano de Estudios Superiores, Zitácuaro, \\ Michoacán, México \\ ${ }^{3}$ Tecnológico de Estudios Superiores de Jocotitlan, \\ Estado de México, México \\ ${ }^{4}$ Universidad Santiago de Cali5, Cali, \\ Valle del Cauca, Colombia \\ fgarciam@uaemex.mx, aafloresf@uaemex.mx, juan_120990@hotmail.com, \\ lage49@yahoo.es, edtore75@hotmail.com, eegrandag@uaemex.mx
}

\begin{abstract}
Resumen. En este trabajo se presenta un Algoritmo Genético de Fertilización in Vitro con codificación real, un método de optimización estocástico soportado teóricamente por las Leyes de la Herencia de Mendel, la Teoría de la Selección de Darwin y los principios de reproducción asistida de Edwards. El algoritmo propuesto es usado en la sintonización de ganancias de los controladores del tipo Proporcional-IntegralDerivativo aplicados en la regulación de voltaje de los capacitores de un convertidor multicelular de 3 células. Los resultados obtenidos son analizados de manera estadística por medio de los tests de Kolmorov, Anova y el post-hoc de Tukey. La técnica propuesta reporta mejores resultados en la reducción del error cuadrático medio en relación con un Algoritmo Genético Canónico y el método de ZieglerNichols.
\end{abstract}

Palabras clave: algoritmo genético con fertilización in vitro, convertidor multicelular, controlador PID. 


\title{
Tuning of PID Controllers by means of a Genetic Algorithm with in vitro Fertilization Applied to a Multicellular Converter
}

\begin{abstract}
In this paper, an In Vitro Fertilization Genetic Algorithm with real coding, a stochastic optimization method theoretically supported by Mendel's laws of inheritance, Darwin's selection theory and Edward's principles of assisted reproduction are presented. The proposed algorithm is used in gain tuning of the Proportional-Integral-Derivative type controllers applied on the voltage regulation of the capacitors of a 3 cell multicellular converter. The results obtained are statistically analized by Kolmorov, Anova and Tukey's post-hoc tests. The proposed technique reports better results of the mean square error reduction than the Canonical Genetic Algorithm and the Ziegler-Nichols method.
\end{abstract}

Keywords: in vitro fertilization genetic algorithm, multicellular converter, PID controller.

\section{Introducción}

Una de las aplicaciones de la electrónica de potencia es la implementación de circuitos electrónicos denominados convertidores multinivel a partir de dispositivos semiconductores de conmutación, diodos y capacitores. Tienen como finalidad la transformación de corriente directa (cd) en corriente alterna (ca) de tal forma que proporcione de manera estable energía a cargas resistivas, capacitivas, inductivas o mixtas. Un ejemplo de esto son los motores de corriente alterna [1]. Una alternativa, que permite el manejo de corrientes elevadas, son los convertidores multicelulares, una serie de arreglos de semiconductores de potencia y capacitores flotados que funcionan como fuentes ideales de voltaje. Una célula de conmutación tiene el esquema que se muestra en la figura 1, donde se puede observar un par de dispositivos de conmutación (Mosfet y Mosfet 1) y un capacitor [2].

En la figura $1 \mathrm{M}_{\mathrm{n}}, \mathrm{M}_{\mathrm{n}}^{\prime}$ y $\mathrm{C}_{\mathrm{n}-1}$ forman la P-ésima célula, la cual está separada por medio de dos capacitores flotados, dado que no se encuentran referenciados a tierra, y que funcionan como fuentes ideales de voltaje. Por lo tanto, un convertidor de $\mathrm{P}_{\mathrm{n}}$ células está constituido por $\mathrm{C}_{\mathrm{n}-1}$ capacitores, cuyos voltajes están determinados por medio de las ecuaciones 1 y 2 :

$$
\begin{gathered}
\mathrm{V}_{\mathrm{T}}=\mathrm{V}_{1}+\mathrm{V}_{2}, \\
\mathrm{~V}_{\mathrm{k}}=\frac{\mathrm{k} * \mathrm{~V}_{\mathrm{T}}}{\mathrm{n}} .
\end{gathered}
$$


donde $\mathrm{V}_{\mathrm{T}}$ es el voltaje de alimentación del convertidor, $\mathrm{k}$ representa la célula y $\mathrm{n}$ el número total de células del convertidor. Con base en lo anterior en [2] y [3] se han diseñado convertidores de 3 células como el mostrado en la figura 3, donde los voltajes presentes en los capacitores son regulados por medio de controladores del tipo Proporcional-IntegralDerivativo (PID), dado que es condición que estos estén estables (con mínimas variaciones) en todo momento.

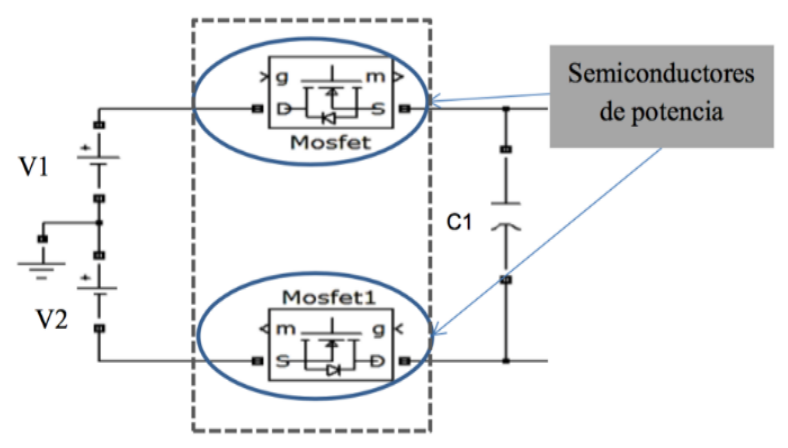

Fig. 1. Célula de conmutación de un convertidor multicelular, formada por dos transistores Mosfet y un capacitor funcionando como fuente flotada.

La topología general de un convertidor multicelular se muestra en la figura 2 donde se ve el conjunto de células de conmutación interconectadas entre sí [3].

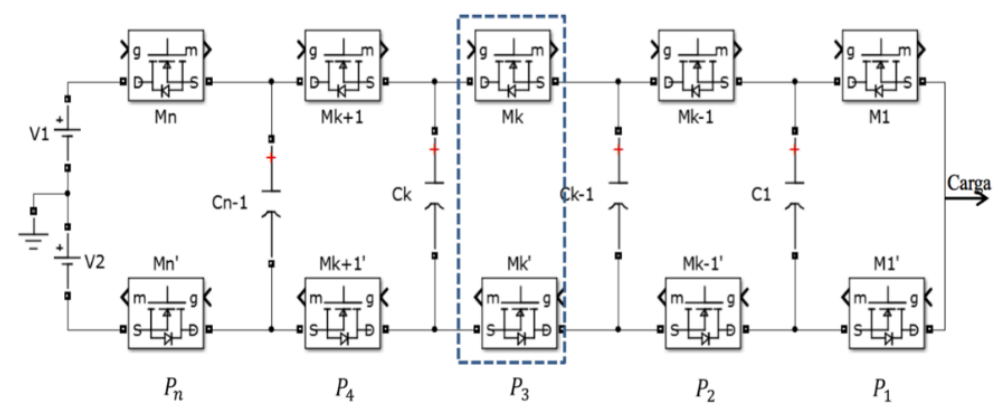

Fig. 2. Un convertidor multicelular de $\mathrm{P}_{\mathrm{n}}$ células, donde se muestra los capacitores necesarios.

Las funciones de transferencia de los controladores PID y de los capacitores se muestran en las ecuaciones 3,4 y 5 respectivamente:

$$
\operatorname{PID}(s)=K_{p}+\frac{K_{i}}{s}+K_{d} s,
$$




$$
\begin{gathered}
\mathrm{C}_{1}(\mathrm{~s})=\frac{0.99596}{0.00002655 \mathrm{~s}^{2}+0.02755 \mathrm{~s}+1}, \\
\mathrm{C}_{2}(\mathrm{~s})=\frac{0.99759}{0.000655 \mathrm{~s}^{2}+0.0518 \mathrm{~s}+1^{\prime}}
\end{gathered}
$$

donde:

$\mathrm{K}_{\mathrm{p}}$ es la ganancia proporcional,

$\mathrm{K}_{\mathrm{i}}$ es la ganancia integral,

$\mathrm{K}_{\mathrm{d}}$ la ganancia derivativa.

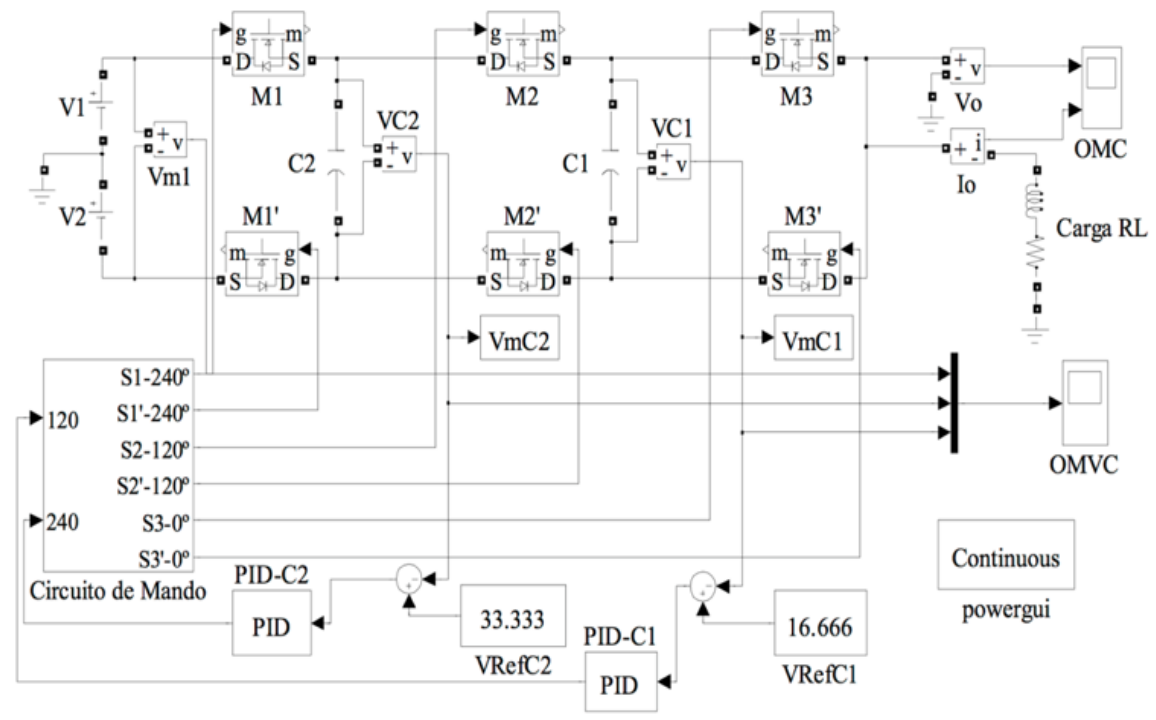

Fig. 3. Diagrama en Simulink ${ }^{\circledR}$ de un convertidor multicelular de 3 niveles con voltaje de los capacitores regulado por medio.

Cabe destacar que en [4] las ganancias de los controladores PID del convertidor multicelular propuesto son sintonizadas fuera de línea, esto por medio de un Algoritmo Genético con codificación real, considerando el problema de sintonización de ganancias como un problema de optimización numérica. Siguiendo este enfoque en este trabajo se propone el ajuste de ganancias de los controladores PID por medio de un método heurístico denominado Algoritmo Genético con Fertilización in Vitro, usando como función objetivo la maximización del inverso del error cuadrático medio dado que es fácil de interpretar analíticamente en relación a otros criterios de desempeño. La finalidad es mejorar los resultados obtenidos de la sintonización por medio de un Algoritmo Genético Canónico y el método de Ziegler Nichols. 


\section{Algoritmo genético con fertilización in vitro}

Junto con el advenimiento de las tecnologías computacionales durante el siglo XX surgieron desarrollos de algoritmos que tuvieran la finalidad de realizar optimizaciones numéricas o combinatorias, una de las primeras investigaciones estuvo a cargo de John Holland profesor de la Universidad de Michigan, el cual propuso uno de los primeros algoritmos evolutivos: El Algoritmo Genético Simple o Canónico (GA, por sus siglas en inglés) [5]. Holland se basó en los trabajos de la Teoría de la Selección Natural de las especies de Charles Darwin, donde se estipula que los miembros de una población tenderán a propagarse en función a su capacidad de adaptación a sus entornos.

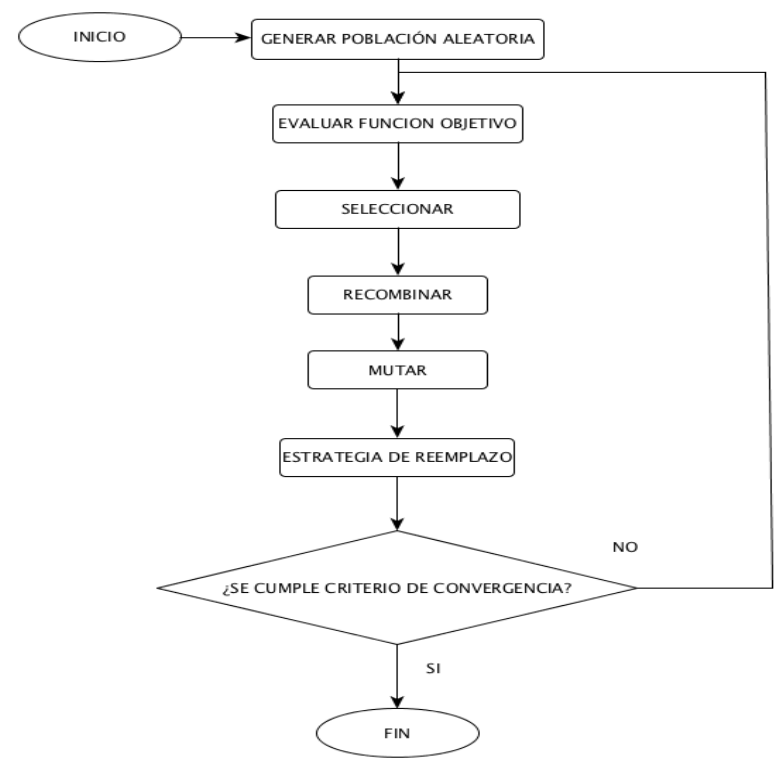

Fig. 4. Diagrama de flujo de un Algoritmo Genético Canónico.

El otro referente teórico de los algoritmos genéticos son las Leyes de la Herencia de Gregory Mendel, las cuales estipulan el carácter hereditario de las características dominantes y recesivas de los individuos que se reproducen sexualmente. Estos preceptos implican que los mejores individuos de una determinada población tienen mayores posibilidades de supervivencia y reproducción; las posibilidades disminuyen o son nulas para los débiles [5]. El GA, creado por John Holland se caracteriza por los pasos descritos en el siguiente pseudocódigo [6,7]:

i. definir una función de aptitud o función objetivo,

ii. generar una serie de posibles soluciones (cromosomas) de manera aleatoria formando un conjunto denominado población, 


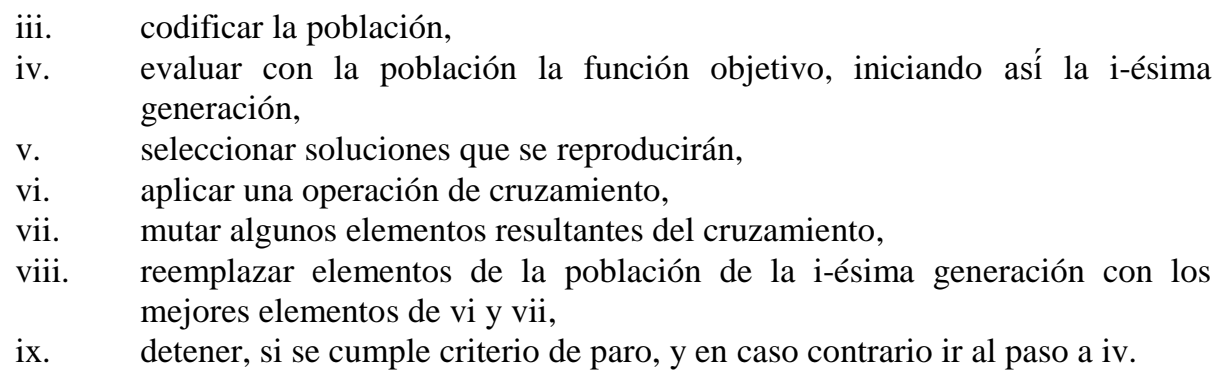

Los pasos descritos se pueden mostrar de forma gráfica en la figura 4.

\subsection{Fertilización in vitro}

En medicina reproductiva existe dos términos que suelen ser tratados en algunas ocasiones como sinónimos, el primero, la esterilidad definida como la incapacidad para concebir, el segundo, la infertilidad es decir la incapacidad de terminar en un producto viable un embarazo. Cabe destacar que a nivel mundial las estadísticas muestran que $20 \%$ de las parejas tienen problemas para embarazarse [9].

Los problemas de salud reproductiva suelen tratarse por medio de diversos procedimientos. Uno de los que presenta mejor índice de éxito es la Fertilización In Vitro (IVF, por sus siglas en inglés) una técnica que empezó a ser desarrollada en la década de los 60's del siglo XX por Edwards (Premio Nobel de Medicina en 2010) a partir de la fecundación en un laboratorio con condiciones controladas de óvulos de ratón para posteriormente ser implantados en una hembra de la misma especie, posteriormente en colaboración con Patric Steptoe escaló la técnicas a parejas de humanos con problemas para concebir, naciendo el primer bebe por IVF en el año 1978 [10]. Los pasos a seguir en una IVF se enlistan a continuación:

- Estimulación: por medio de ciertos medicamentos se estimula la maduración de células reproductivas femeninas (óvulos),

- Recuperación: cuando las células están maduras estas son extraídas por medio de una punción realizada por laparoscopia,

- Fecundación los óvulos son fecundados por células sexuales masculinas (espermas) en un ambiente contralado, con la finalidad de generar embriones viables,

- Implantación: al menos un embrión es implantado en el útero del espécimen femenino el cual idealmente conduciría a una prueba de embarazo exitosa.

\subsection{Pseudocódigo de un algoritmo genético con fertilización in vitro}

Las investigaciones realizadas por Edwards, fundamentaron el diseño de un nuevo método heurístico denominado Algoritmo Genético con Fertilización In Vitro (IVF/GA, 
por sus siglas en inglés) realizado por Camilo Jr. Un investigador del Instituto de Informática de la Universidad Federal de Gois. En esta nueva técnica se explora la posibilidad de usar los cromosomas que no fueron seleccionados para recombinación por medio de analogías con el proceso de reproducción asistida descrito en la sección 2.1.

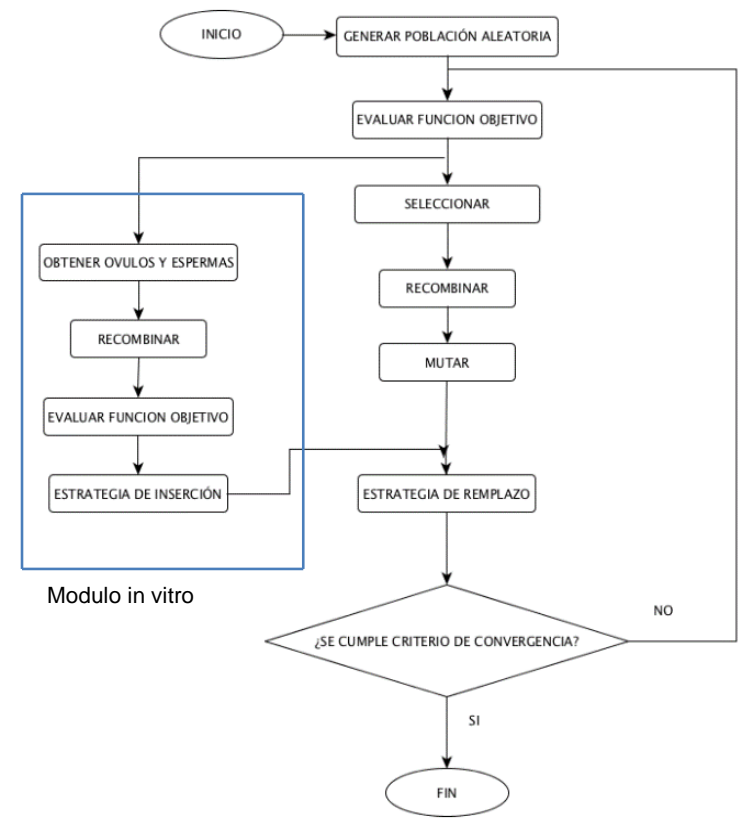

Fig. 5. Diagrama de flujo del IVF/GA propuesto en este trabajo, en azul se observa el módulo de Fertilización In Vitro.

En sus orígenes la técnica se basaba en la codificación binaria de los cromosomas que forman la población del algoritmo. La propuesta que se presenta en este trabajo consiste en cambiar las cadenas binarias por vectores $\vec{x} \in \mathbb{R}$. Esto permite solucionar problemas de optimización numérica de manera más eficiente en relación con codificaciones binarias donde los genes $g \in \mathbb{Z}$. Los pasos del algoritmo IVF/GA que se propone en este artículo basado en [8] se muestran a continuación:

i. definir una función de aptitud o función objetivo,

ii. generar una serie de posibles soluciones (cromosomas) de manera aleatoria

iii. formando un conjunto

iv. evaluar con la población, la función objetivo iniciando así la i-ésima generación,

v. seleccionar soluciones que se reproducirán, 
vi. del porcentaje de la población que no se seleccionan para recombinación se etiquetan como espermas,

vii. el mejor individuo de la generación se usa como óvulo,

viii. recombinar espermas y óvulos generando una serie de soluciones denominadas embriones,

ix. evaluar gametos en la función objetivo,

x. Recombinar los cromosomas resultantes de la sección v,

xi. mutar algunos cromosomas resultantes de $\mathrm{x}$,

xii. reemplazar elementos de la población de la i-ésima generación con los mejores elementos de ix, x y xi,

xiii. detener, si se cumple criterio de paro, y en caso contrario ir al paso a iv.

La figura 5 muestra el diagrama de flujo de un Algoritmo Genético con Fertilización In Vitro (IVF/GA por sus siglas en inglés) y resalta el modulo in vitro.

\section{Metodología}

Se puede estipular que existen dos sistemas de control a lazo cerrado, ambos basados en un PID cuyas ganancias son sintonizadas por medio de GA's y IVF/GA's descritos en la sección 2. La ecuación 6 muestra el cálculo de la función de transferencia para los sistemas propuestos. Por otra parte, la ecuación 7 muestra la función objetivo a maximizar por los algoritmos mencionados

$$
\begin{gathered}
V c_{n}(s)=\frac{P I D_{n}(s) C_{n}(s)}{1+P I D_{n}(s) C_{n}(s)} * \operatorname{Vref}_{n}, \\
\operatorname{fobj}_{n}\left(K_{p}^{n}, K_{i}^{n}, K_{d}^{n}\right)=\max \left(\frac{1}{1+\sqrt{\frac{1}{T} \int_{0}^{T} E\left(K_{p}^{n}, K_{i}^{n}, K_{d}^{n}\right)^{2}}}\right)
\end{gathered}
$$

donde:

$V c_{n}(s)$ representa el voltaje del n-ésimo capacitor,

$C_{n}(s)$ es la función de transferencia del n-ésimo capacitor,

$P I D_{n}(s)$ la función de transferencia del n-ésimo controlador PID,

Vre $f_{n}$ es el voltaje de referencia del n-ésimo sistema de control,

$\operatorname{fobj}_{n}\left(K_{p}^{n}, K_{i}^{n}, K_{d}^{n}\right)$ es la función objetivo del n-ésimo sistema de control, 
$\frac{1}{T} \int_{0}^{T} E\left(K_{p}^{n}, K_{i}^{n}, K_{d}^{n}\right)^{2}$ es el error cuadrático medio del n-ésimo sistema de control en términos de las ganancias del control PID,

$T$ es el tiempo de simulación,

n es el número de sistemas de control usados el cual es P-1 células.

Tabla 1. Condiciones de generación de cromosomas.

\begin{tabular}{lc}
\hline Cromosoma de $\mathrm{Vc}_{1}$ & Cromosoma de $\mathrm{Vc}_{2}$ \\
\hline $\mathrm{K}_{\mathrm{p}}=(30+(\operatorname{random}[0,1]) 60)$ & $\mathrm{K}_{\mathrm{p}}=(2+(\operatorname{random}[0,1]) * 30)$ \\
$\mathrm{K}_{\mathrm{i}}=((\operatorname{random}[0,1]) 10000)$ & $\mathrm{K}_{\mathrm{i}}=(1000+(\operatorname{random}[0,1]) * 1000)$ \\
$\mathrm{K}_{\mathrm{p}}=((\operatorname{random}[0,1]) * 0.01)$ & $\mathrm{K}_{\mathrm{p}}=((\operatorname{random}[0,1]) * 0.9)$ \\
\hline
\end{tabular}

Como se ha expuesto anteriormente el problema de diseño de los controladores PID de un convertidor multicelular de tres células se divide en el estudio de dos sistemas de control con sus correspondientes funciones objetivo. Esto implica la generación de dos diferentes espacios de búsqueda (colección de cromosomas) formados por vectores de números reales con el siguiente formato:

$$
\text { cromosoma }=\left[k_{p}, k_{i}, k_{d}\right]^{T} \text {. }
$$

La tabla 1 muestra las condiciones de generación de los cromosomas para cada uno de los sistemas de control, los cuales son identificados como $V c_{1}$ y $V c_{2}$

Los cromosomas que se seleccionaron para el cruzamiento en sucesivas generaciones fueron escogidos mediante un operador de ruleta, dado que este favorece y preserva el carácter estocástico de los algoritmos genéticos.

El operador de cruce propuesto para el GA y IVF/GA es el BLX- $\propto$, (mostrado en la ecuación 8), este es usado dado que permite una exploración fuera de los límites impuestos por los cromosomas a recombinar a través de la generación aleatoria de los descendientes con distribución una distribución de probabilidad uniforme [11]:

$$
\mathrm{C}^{\mathrm{H}}=\operatorname{rand}\left[\left(\mathrm{h}_{\min }-\mathrm{I} * \alpha\right),\left(\mathrm{h}_{\max }+\mathrm{I} * \alpha\right)\right] \text {. }
$$

Donde $\mathrm{C}^{\mathrm{H}}$ es el cromosoma descendiente $\mathrm{h}_{\min }=\min \left(\mathrm{C}_{\mathrm{i}}^{1}, \mathrm{C}_{\mathrm{i}}^{2}\right), \mathrm{h}_{\max }=\max \left(\mathrm{C}_{\mathrm{i}}^{1}, \mathrm{C}_{\mathrm{i}}^{2}\right), \mathrm{I}=$ $\mathrm{h}_{\max }-\mathrm{h}_{\min }, \alpha=\operatorname{rand}[0,1]$ con distribución uniforme.

La mutación se realiza por medio de un operador gaussiano. A partir de un cromosoma $\mathrm{C}_{\mathrm{i}}^{\prime}$ se puede obtener un cromosoma transformado o mutado $\mathrm{C}_{\mathrm{i}}^{\prime \prime}$ a partir de la siguiente expresión donde el tamaño de paso de la mutación $\beta=[0,1]$ y la dirección de la misma se representa por d (ecuación 9) [11]:

$$
C_{i}^{\prime \prime}=C_{i}^{\prime}+\beta * d .
$$


J. F. García-Mejía, A. A. Flores-Fuentes, J. C. Ambriz-Polo, L. A. González-Escobar, et al.

Tabla 2. Experimentos a realizar.

\begin{tabular}{lllllllc}
\hline Test & Técnica & Cromosomas & $\begin{array}{l}\text { Porcentaje } \\
\text { Cruzamiento }\end{array}$ & $\begin{array}{l}\text { Porcentaje } \\
\text { Mutación }\end{array}$ & Óvulos & Espermas & $\begin{array}{l}\text { Sistema } \\
\text { de Control }\end{array}$ \\
\hline 1 & GA & 100 & $80 \%$ & $1 \%$ & -- & -- & $\mathrm{Vc}_{1}$ \\
2 & GA & 100 & $60 \%$ & $1 \%$ & -- & -- & $\mathrm{Vc}_{1}$ \\
3 & IVF/GA & 100 & $80 \%$ & $1 \%$ & 1 & 20 & $\mathrm{Vc}_{1}$ \\
4 & IVF/GA & 100 & $60 \%$ & $1 \%$ & 1 & 20 & $\mathrm{Vc}_{1}$ \\
5 & GA & 100 & $80 \%$ & $1 \%$ & --- & & $\mathrm{Vc}_{2}$ \\
6 & GA & 100 & $60 \%$ & $1 \%$ & --- & & $\mathrm{Vc}_{2}$ \\
7 & IVF/GA & 100 & $80 \%$ & 1 & 1 & 20 & $\mathrm{Vc}_{2}$ \\
8 & IVF/GA & 100 & $60 \%$ & 1 & 1 & 20 & $\mathrm{Vc}_{2}$ \\
\hline
\end{tabular}

A partir del anterior se plantea una serie de test que se describen en la tabla 2 los cuales suelen se consideraron a partir de los datos de fertilidad, es decir entre un 40 y un $20 \%$ de las parejas tienen problemas reproductivos. Los resultados de los experimentos se muestran en la sección 4. La finalidad es demostrar la pertinencia de algoritmo IVF/GA, como un método de sintonización de ganancias de controladores aplicados a un convertir de energía multicelular.

Cada uno de los tests o experimentos se ejecutan 35 veces, esto permite analizar estadísticamente la repetitividad es decir la estabilidad del algoritmo. Estos estudios también permiten describir si existen diferencias estadísticamente significativas entre los experimentos realizados

\section{Resultados}

Los resultados reportados en esta sección son expresados en términos del error cuadrático medio de cada sistema de control. En las figuras 6 (a) y 6 (b) se muestra la convergencia de los GA (color azul) y IVF/GA (rojo) para el sistema de control $\mathrm{Vc}_{1}$ con una posibilidad de cruzamiento del $60 \%$ y $80 \%$ respectivamente. Los resultados con el porcentaje de cruzamiento del $60 \%$ y $80 \%$ son mostrados en las figuras 7 (a) y 7 (b) para el sistema de control $\mathrm{Vc}_{2}$.

Se puede observar que la convergencia no presenta diferencias significativas entre ambas técnicas para los dos sistemas de control que componen el convertidor muticelular propuesto.

Por otra parte la tabla 3 muestra los resultados estadísticos realizados sobre 35 ejecuciones, lo cual permite determinar cuál algoritmo cumple con los mejores criterios de estabilidad a través del cálculo de la repetitividad (desviación estándar entre media aritmética). Además se aplicó un test de Kolmorov-Smirnov, (con la finalidad comprobar el comportamiento estocástico de los algoritmo propuestos). 
La tabla 3 muestra, de acuerdo al test de normalidad de Kolmorov-Smirnov (K-S), que todos los experimentos tienen un comportamiento estocástico. A partir de esto se plantea un test de Anova con la finalidad de determinar si existen diferencias estadísticamente significativas entre los experimentos desarrollados en este trabajo. Los resultados se muestran en la tabla 4.

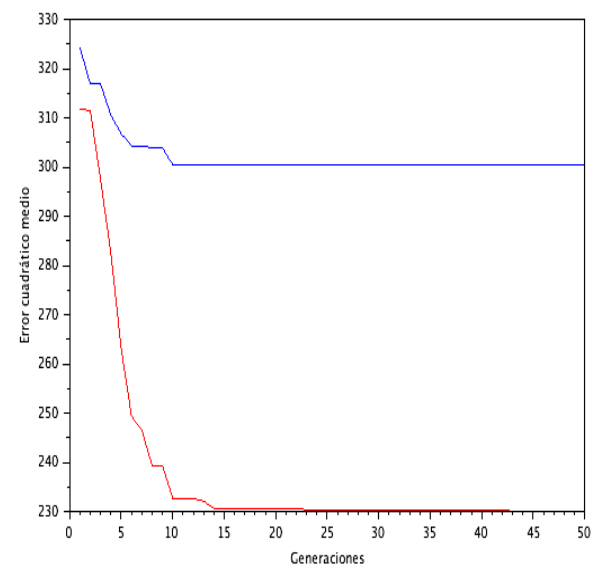

(a)

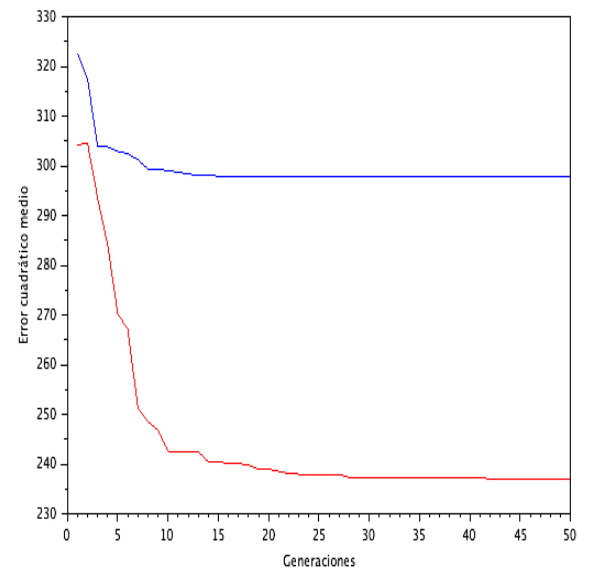

(b)

Fig. 6. Convergencia de los ajustes de los sistemas $\mathrm{Vc}_{1}$.

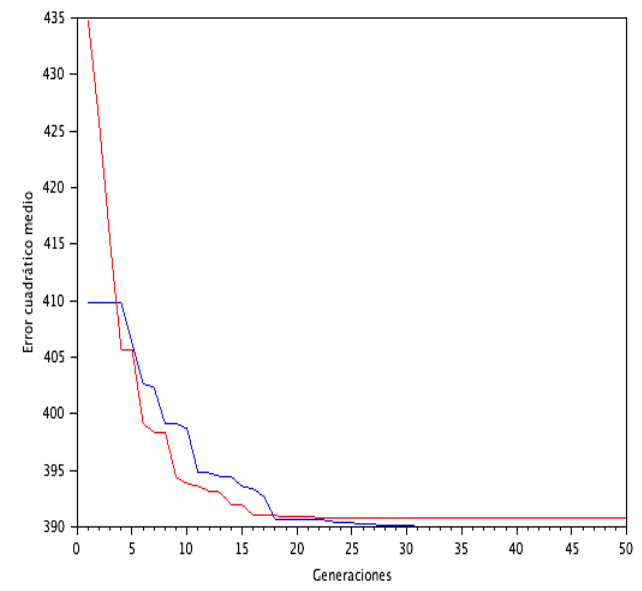

(a)

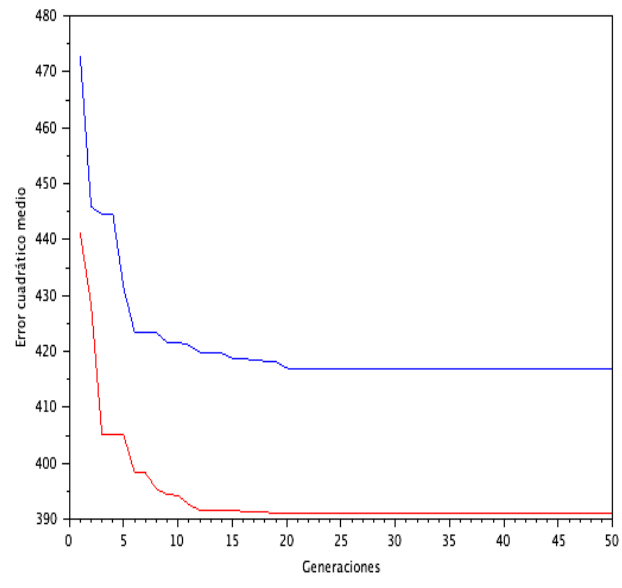

(b)

Fig. 7. Convergencia de los ajustes de los sistemas $\mathrm{Vc}_{2}$. 
J. F. García-Mejía, A. A. Flores-Fuentes, J. C. Ambriz-Polo, L. A. González-Escobar, et al.

Tabla 3. Estadísticos de los experimentos de los sistemas de control $\mathrm{Vc}_{1} \mathrm{y} \mathrm{Vc}_{2}$.

\begin{tabular}{cccccc}
\hline Test & Técnica & $\begin{array}{c}\text { Valor } \\
\text { Medio }\end{array}$ & $\begin{array}{c}\text { Desviación } \\
\text { Estándar }\end{array}$ & Repetitividad & $\begin{array}{c}\text { Significancia } \\
\text { asintótica (K-S) }\end{array}$ \\
\hline 1 & GA & 301.9177 & 10.6245 & 3.5190 & 0.980 \\
2 & GA & 305.3213 & 11.0000 & 3.6027 & 0.820 \\
3 & IVF/GA & 264.6754 & 27.2457 & 10.2940 & 0.270 \\
4 & IVF/GA & 269.2317 & 28.8980 & 10.7335 & 0.660 \\
5 & GA & 398.1020 & 7.5952 & 1.907 & 0.304 \\
6 & GA & 401.4855 & 10.4917 & 2.6132 & 0.508 \\
7 & IVF/GA & 390.5473 & 7.4637 & 1.9111 & 0.156 \\
8 & IVF/GA & 392.8595 & 7.2456 & 1.8443 & 0.685 \\
\hline
\end{tabular}

Tabla 4. Análisis de Anova de los experimentos.

\begin{tabular}{|c|c|c|c|c|c|c|}
\hline & & $\begin{array}{c}\text { Suma } \\
\text { de } \\
\text { Cuadrados }\end{array}$ & $\begin{array}{l}\text { Grados de } \\
\text { libertad }\end{array}$ & $\begin{array}{c}\text { Media } \\
\text { cuadrática }\end{array}$ & S & $\begin{array}{l}\text { Significa } \\
\text { ncia }\end{array}$ \\
\hline \multirow{3}{*}{$\begin{array}{l}\text { Valor de la función } \\
\text { Objetivo }\end{array}$} & $\begin{array}{l}\text { Entre } \\
\text { Grupos }\end{array}$ & 904335.333 & 7 & 129190.762 & \multirow{3}{*}{495.15} & \multirow{3}{*}{153} \\
\hline & $\begin{array}{c}\text { Intra } \\
\text { Grupos }\end{array}$ & 70967.681 & 272 & \multirow[t]{2}{*}{260.911} & & \\
\hline & Total & $\begin{array}{c}975303.01 \\
4\end{array}$ & 279 & & & \\
\hline
\end{tabular}

Las tablas 5 y 6 muestran los resultados del test de Tukey sobre los experimentos propuestos para los sistemas de control $\mathrm{Vc}_{1} \mathrm{y} \mathrm{Vc}_{2}$ respectivamente, colocando en color rojo el valor correspondiente al menor valor del error cuadrático medio.

Tabla 5. Test de Tukey para experimentos sobre el sistema $\mathrm{Vc}_{1}$.

\begin{tabular}{ccc}
\hline & \multicolumn{2}{c}{ Subconjuntos para alpha $=0.05$} \\
\hline Experimento & 1 & 2 \\
1 & 301.9177 & \\
2 & 305.3213 & \\
3 & & 264.6754 \\
4 & & 269.2317 \\
\hline
\end{tabular}

En la tabla 5 se muestra que los resultados de los experimentos 1 y 2, que fueron sujetos de optimización por medio de un GA, son estadísticamente similares. Por otra parte la media de los experimentos 3 y 4, los cuales fueron tratados con el IVF/GA propuesto en este 
artículo son similares entre sí. Por otra parte, la tabla 6, representativa del sistema de control $\mathrm{Vc}_{2}$ tienen un comportamiento similar a $\mathrm{Vc}_{1}$.

Tabla 6. Test de Tukey para experimentos sobre el sistema $\mathrm{Vc}_{2}$.

\begin{tabular}{ccc}
\hline & \multicolumn{2}{c}{ Subconjuntos para alpha $=0.05$} \\
\hline Experimento & 1 & 2 \\
5 & 398.1020 & \\
6 & 401.4855 & \\
7 & & 390.5473 \\
8 & & 392.8595 \\
\hline
\end{tabular}

Las tablas 5 y 6 muestran que los errores cuadráticos medios de los sistemas $V_{c_{1}}$ y $V_{c_{2}}$ son menores cuando se ajustan las ganancias de sus PID's con un IVF/GA en comparación con el GA.

Las figuras 8 (a) y 8 (b) muestran los voltajes de salida de los capacitores $\mathrm{C}_{1}$ y $\mathrm{C}_{2}$ respectivamente, los cuales son obtenidos por la acción de los PID's ajustados por un IVF/GA (color rojo) y GA (en color azul) con una probabilidad de cruce del $80 \%$, dado los resultados reportados. En las mismas figuras también se compara con el ajuste de ZieglerNichols (negro).

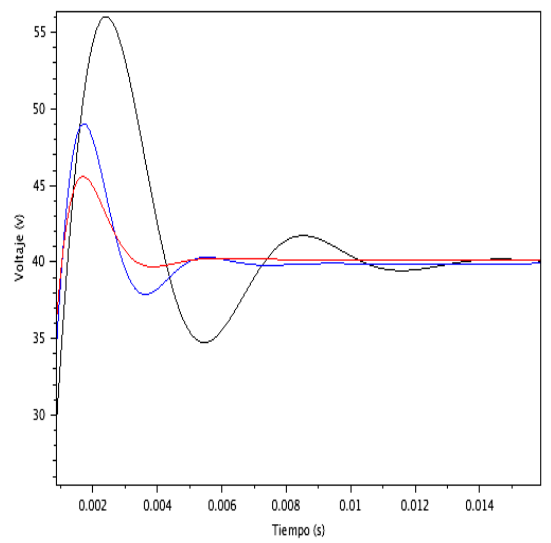

(a)

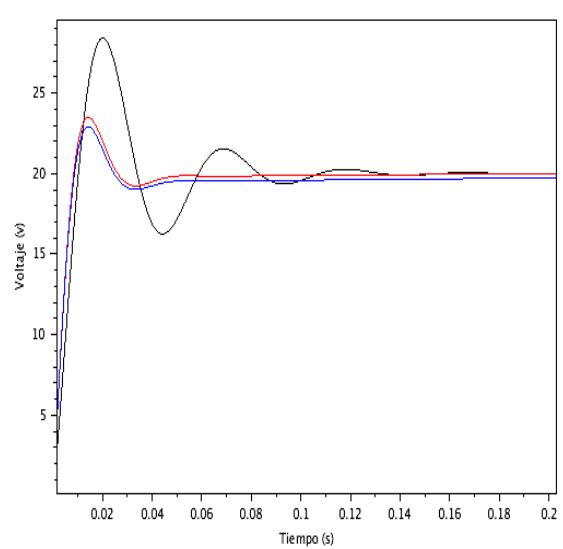

(b)

Fig. 8. Voltajes de los capacitores $C_{1}$ y $C_{2}$ regulados con PID'd.

La tabla 7 muestra un ejemplo de las ganancias de los controladores, las cuales son obtenidas por las técnicas que presentan menor valor de error cuadrático medio. La tabla 8 muestra los índices de desempeño que permite valorar la acción de los controladores diseñados en esta propuesta. 
J. F. García-Mejía, A. A. Flores-Fuentes, J. C. Ambriz-Polo, L. A. González-Escobar, et al.

Tabla 7. Valores de las ganancias PID de los sistemas $\mathrm{Vc}_{1}$ y $\mathrm{Vc}_{2}$.

\begin{tabular}{lcccc}
\hline \multirow{2}{*}{ Sistema } & $\begin{array}{c}\text { Técnica } \\
\text { empleada }\end{array}$ & $\mathrm{K}_{\mathrm{p}}$ & $\mathrm{K}_{\mathrm{i}}$ & $\mathrm{K}_{\mathrm{d}}$ \\
\hline \multirow{3}{*}{$\mathrm{Vc}_{1}$} & $\mathrm{GA}$ & 89.11892 & 2257.9539 & 0.0155993 \\
& $\mathrm{IVF}$ & 88.789632 & 5236.8697 & 0.0308438 \\
& Ziegler & 51.037022 & 33073.821 & 0.0196891 \\
& Nichols & 25.006147 & 107.51478 & 0.0943677 \\
$\mathrm{Vc}_{2}$ & $\mathrm{GA}$ & 25.393505 & 309.03829 & 0.0940209 \\
& IVF & 14.79555 & 1032.4077 & 0.0530092 \\
\hline
\end{tabular}

Tabla 8. Criterios de desempeño de los sistemas $\mathrm{Vc}_{1}$ y $V c_{2}$.

\begin{tabular}{|c|c|c|c|c|c|}
\hline Sistema & $\begin{array}{c}\text { Técnica } \\
\text { empleada }\end{array}$ & Error RMS & $\begin{array}{c}\text { Porcentaje de } \\
\text { sobre impulso }\end{array}$ & $\begin{array}{c}\text { Tiempo de } \\
\text { establecimiento }\end{array}$ & $\begin{array}{c}\text { Error estado } \\
\text { estable }\end{array}$ \\
\hline \multirow{3}{*}{$\mathrm{Vc}_{1}$} & $\mathrm{GA}$ & 305.79742 & 22.6301 & 0.007 & 0.06174 \\
\cline { 2 - 6 } & IVF & 264.74921 & 13.997 & 0.007 & -0.01 \\
\cline { 2 - 6 } & $\begin{array}{c}\text { Ziegler } \\
\text { Nichols }\end{array}$ & 389.82819 & 40.1245 & 0.02 & -0.000008 \\
\hline \multirow{3}{*}{$\mathrm{Vc}_{2}$} & GA & 388.4005 & 14.5610 & 0.06 & 1.1120 \\
\cline { 2 - 6 } & IVF & 387.57442 & 17.4101 & 0.05 & 0.0862 \\
\cline { 2 - 6 } & $\begin{array}{c}\text { Ziegler } \\
\text { Nichols }\end{array}$ & 587.93244 & 42.0535 & 0.16 & 0.00685 \\
\hline
\end{tabular}

\section{Conclusiones}

A partir de las pruebas estadísticas mostradas se puede observar que el Algoritmo Genético con Fertilización In Vitro presenta las siguientes ventajas:

a) De acuerdo a la Tabla 3, para los sistemas de control $\mathrm{Vc}_{1}$ y $\mathrm{Vc}_{2} \operatorname{los}$ menores errores cuadráticos medios son obtenidos con el IVF/GA.

b) Convergencia no se ve afectada.

c) La repetitividad obtenida por el IVF/GA es menor con respecto con el GA.

Las tablas 5 y 6 muestran que los experimentos realizados con el GA son estadísticamente iguales. Se observa, de acuerdo al test de Tukey, que el IVF/GA es una técnica diferente a un algoritmo Genético Canónico y nueva de acuerdo a lo revisado en la literatura especializada. Se demuestra que los cromosomas con baja aptitud al recombinarse 
con un cromosoma de valor alto inciden en la convergencia del algoritmo. Es necesario explorar los efectos de los operadores de cruzamiento en el algoritmo IVF/GA, esto puede modificar la repetitividad.

Como trabajo a futuro se plantea el diseño otros esquemas de regulación de voltaje de los capacitores flotados de un convertidor multicelular tales como el control difuso y las Redes Neuronales Artificiales.

\section{Referencias}

1. Gazzam, N., Benalia A: Observability Analysis and Observer Design of Multicellular Converters. In: $8^{\text {th }}$ International Conference on Modelling, Identification and Control (ICMIC2016), IEEE, Algiers, Algeria (2016)

2. Rossano Díaz, I. O.: Sistema digital aplicado a un convertidor multinivel estático. Tesis de maestría en Ciencias en Ingeniería Electrónica, Instituto Tecnológico de Toluca, Instituto Nacional de Investigaciones Nucleares, Toluca, Estado de México, México (2009)

3. Flores Fuentes, A. A.: Generador de RF de alta potencia por medio de un inversor multinivel. Tesis de Doctorado en Ciencias en Ingeniería Electrónica, Instituto Tecnológico de Toluca, Instituto Nacional de Investigaciones Nucleares, Toluca, Estado de México, México (2009)

4. Flores Fuentes A. A.: Algoritmo Genético implementado en la optimización de los parámetros de un controlador PID aplicado a un convertidor estático de energía. En: Congreso Internacional de Ingeniería Electrónica Electro, pp. 89-94, Instituto Tecnológico de Chihuahua, Chihuahua, México (2013)

5. Yang, X. S.: Nature-Inspired Metaheuristic Algorithms. United Kingdom: Luniver Press (2011)

6. Mitchell, M.: An introduction to genetic algorithms. MIT Press, USA (1998)

7. Gen, M., Cheng, R.: Genetic algorithms and engineering optimization. John Wiley and Sons (2000)

8. Camilo, C., Yamanaka, K.: In Vitro Fertilization Genetic Algorithm Applied to Multidimensional 0-1 Knapsack Problem. In: Proceedings of the 2011 World Congress in Computer Science, Computer Engineering, and Applied Computing (2011)

9. Oliva, R.: Genética Humana. 3er edn. Edicions Universitat Barcelona (2004)

10. Elder, K.: In Vitro Fertilization. 3er edn Cambridge University Press, United Kingdom (2010)

11. Takahashi, M.: A Crossover Operator Using Independent Component Analysis for Real-Coded Genetic Algorithms. In: Proceedings of the 2011 IEEE Congress on Evolutionary Computation (2011) 